\title{
Improving the $Q$ Factor of an Optical Atomic Clock Using Quantum Nondemolition Measurement
}

\author{
William Bowden $\odot,{ }^{1, *}$ Alvise Vianello๑, ${ }^{1,2, *}$ Ian R. Hillø, ${ }^{1}$ Marco Schioppo๑, ${ }^{1}$ and Richard Hobson $\odot^{1}$ \\ ${ }^{1}$ National Physical Laboratory, Hampton Road, Teddington TW11 OLW, United Kingdom \\ ${ }^{2}$ Blackett Laboratory, Imperial College London, Prince Consort Road, London SW7 2AZ, United Kingdom
}

(Received 24 July 2020; revised 20 September 2020; accepted 13 October 2020; published 15 December 2020)

\begin{abstract}
Quantum nondemolition (QND) measurement is a remarkable tool for the manipulation of quantum systems. It allows specific information to be extracted while still preserving fragile quantum observables of the system. Here we apply cavity-based QND measurement to an optical lattice clock-a type of atomic clock with unrivaled frequency precision - preserving the quantum coherence of the atoms after readout with $80 \%$ fidelity. We apply this technique to stabilize the phase of an ultrastable laser to a coherent atomic state via a series of repeated QND measurements. We exploit the improved phase coherence of the ultrastable laser to interrogate a separate optical lattice clock, using a Ramsey spectroscopy time extended from $300 \mathrm{~ms}$ to $2 \mathrm{~s}$. With this technique we maintain $95 \%$ contrast and observe a sevenfold increase in the clock's $Q$ factor to $1.7 \times 10^{15}$.
\end{abstract}

DOI: 10.1103/PhysRevX.10.041052

Subject Areas: Atomic and Molecular Physics

\section{INTRODUCTION}

In quantum nondemolition (QND) measurement, an observable $\hat{S}$ of a quantum system is coupled to an observable $\hat{M}$ of a "meter" system, so that direct measurement of $\hat{M}$ yields indirect information about $\hat{S}$. While the measurement of $\hat{M}$ may perturb the state of the meter, the inferred value of the observable $\hat{S}$ is conserved by the QND measurement [1]. QND measurements have given us a window on a wide range of quantum systems, including circuit quantum electrodynamics [2-4], solid-state spin qubits [5-7], mechanical oscillators [8,9], photons [10-13], nitrogen-vacancy centers [14], and trapped ions [15,16].

In this work we use QND measurement to observe cold $\mathrm{Sr}$ atoms in an optical lattice clock (OLC), in pursuit of metrological enhancements already demonstrated in $\mathrm{Rb}$ and Cs-based magnetometers $[17,18]$ and microwave atomic clocks [19-23]. Our work builds on recent demonstrations with $\mathrm{Yb}$ [24] and $\mathrm{Sr}[25,26]$ by applying QND measurement to a fully operational Sr OLC - an exceptionally stable and accurate type of clock [27-30] which is a prime candidate to underpin a future redefinition of the second in the International System of Units [31] as well as being a sensitive probe for geodesy [32,33] and physics beyond the standard model [34-37].

\footnotetext{
"These authors contributed equally to this work.
}

Published by the American Physical Society under the terms of the Creative Commons Attribution 4.0 International license. Further distribution of this work must maintain attribution to the author(s) and the published article's title, journal citation, and DOI.
The OLC works by steering the frequency of an ultrastable laser, or "local oscillator" (LO), to match the frequency of the optical ${ }^{1} S_{0}{ }^{3} P_{0}$ clock transition in atomic $\mathrm{Sr}$. The LO frequency is initialized close to resonance with the atomic clock transition, then a spectroscopy pulse is carried out on $\mathrm{Sr}$ atoms confined in an optical lattice in the ${ }^{1} S_{0}$ ground state. At the end of the spectroscopy pulse, the frequency detuning between the LO and the atomic resonance is inferred by measuring the fraction of atoms excited into the ${ }^{3} P_{0}$ state. In earlier realizations of the OLC [27-30] the excitation fraction is measured using fluorescence detection, which destroys the atomic sample. Stabilization of the LO therefore requires new atomic samples to be prepared, interrogated, and measured in a repeated cycle. By contrast, in this work the excitation fraction is measured using QND methods, allowing the atoms to be recycled for another spectroscopy pulse immediately after measurement.

We carry out QND measurement in an OLC by surrounding the $\mathrm{Sr}$ atoms with a high-finesse optical cavity at $461 \mathrm{~nm}$, the wavelength of the strong ${ }^{1} S_{0^{-}}{ }^{1} P_{1}$ transition. The same optical cavity also supports a magic-wavelength optical lattice trap [38]. The $461 \mathrm{~nm}$ intracavity photons serve as a QND meter of the number of ground state atoms, experiencing a measurable phase shift due to dispersion from the ${ }^{1} S_{0^{-}}{ }^{1} P_{1}$ transition. In this work we demonstrate that, for short probe times, the QND measurement is weak and therefore preserves with high fidelity the coherence of atoms prepared in a superposition of ${ }^{1} S_{0}$ and ${ }^{3} P_{0}$. This nondestructive detection enables operation of the OLC in new, more stable configurations, such as the "atom phase lock" (APL), in which the phase of the LO is stabilized to the phase evolution of the atoms. Here we show that the APL significantly 
improves the coherence time of the LO laser. Deploying the phase-locked LO in a second, cointerrogated OLC enables us to extend the Ramsey dark time $T$, thereby reducing the Fourier-limited linewidth of the atomic signal $\Delta \nu=1 /(2 T)$. This leads to an increased $Q$ factor-i.e., an increased ratio $Q=\nu_{0} / \Delta \nu$ between the clock transition frequency $\nu_{0}$ and the spectroscopic linewidth-enhancing a key figure of merit impacting the measurement precision of the clock.

\section{QUANTUM NONDEMOLITION MEASUREMENT IN AN OPTICAL LATTICE CLOCK}

To operate the OLC, fermionic strontium $\left({ }^{87} \mathrm{Sr}\right)$ is laser cooled and loaded into a magic-wavelength, onedimensional optical lattice at $813 \mathrm{~nm}$. A Ramsey spectroscopy sequence then maps phase or frequency errors of the LO, in this case an ultrastable laser at $698 \mathrm{~nm}$ (see Supplemental Material [39]), onto a population imbalance between the electronic ground state $|g\rangle\left(5 s^{2}{ }^{1} S_{0}\right.$, $\left.M_{F}= \pm 5 / 2\right)$ and the long-lived excited state $|e\rangle(5 s 5 p$ ${ }^{3} P_{0}, M_{F}^{\prime}= \pm 3 / 2$ ). Adopting a pseudospin formulation, this population imbalance is encoded in the observable $\hat{S}_{z}$, the $z$ component of the collective spin of the system $\hat{S}$. The collective spin components can be defined as

$$
\begin{gathered}
\hat{S}_{x}=\frac{1}{2}\left(\hat{S}_{g e}+\hat{S}_{e g}\right), \\
\hat{S}_{y}=\frac{1}{2 i}\left(\hat{S}_{e g}-\hat{S}_{g e}\right), \\
\hat{S}_{z}=\frac{1}{2}\left(\hat{S}_{e e}-\hat{S}_{g g}\right),
\end{gathered}
$$

where the operators $\hat{S}_{i j}=\sum_{k=1}^{N}|i\rangle_{k}|j\rangle_{k}$ are summed over all atoms in the sample.

For a typical OLC, $S_{z}$ is measured destructively in a twostep process [27-30]. First, a strong transition at $461 \mathrm{~nm}$ from the ground state to an auxiliary state $\left(5 s 5 p^{1} P_{1}\right)$ is used to measure $S_{g g}$ via fluorescence detection. The fluorescence pulse heats the ground state atoms, causing them to escape from the lattice. Next, excited state atoms are optically pumped into the ground state and the fluorescence detection is repeated, giving a measurement
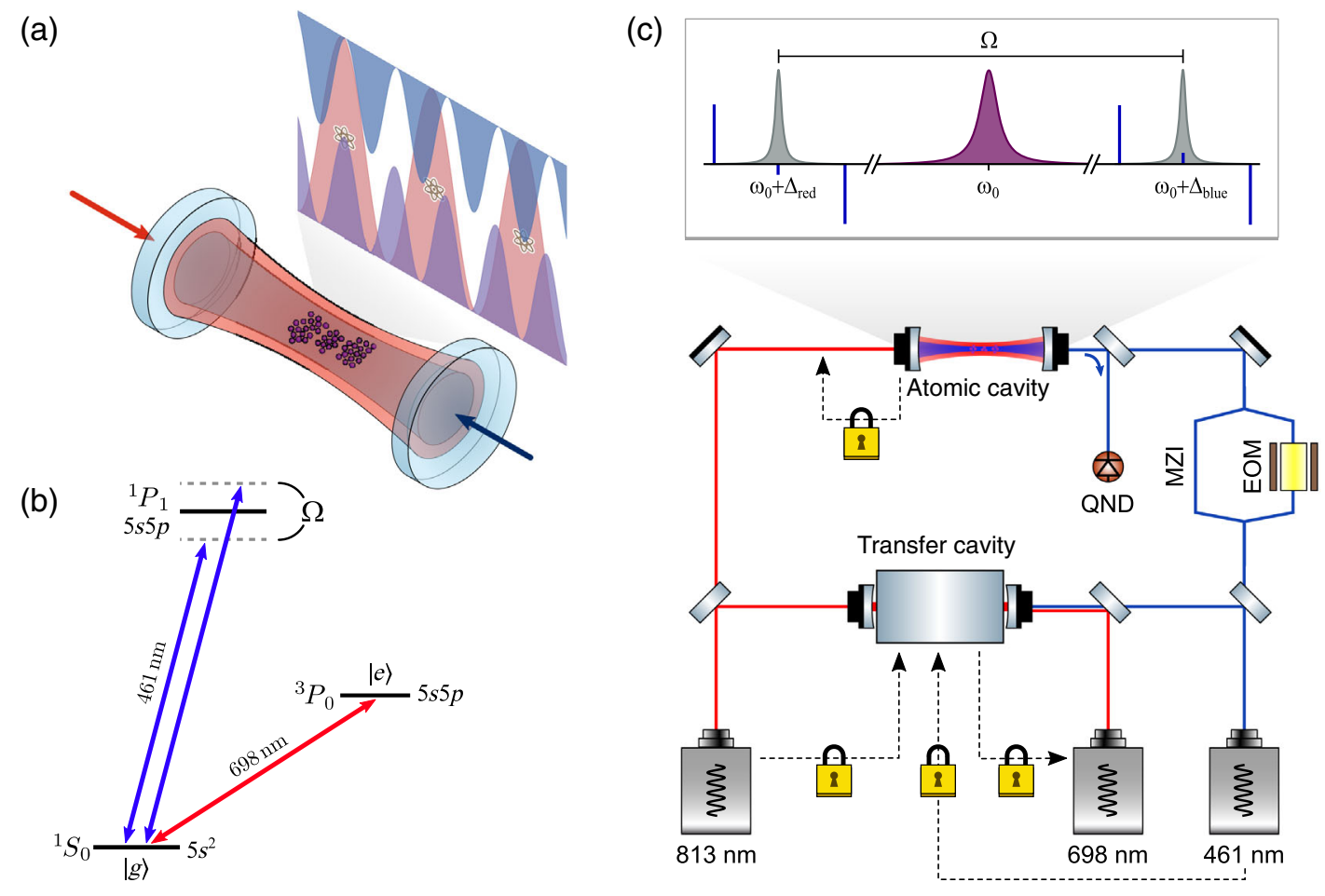

FIG. 1. Overview of the QND measurement scheme. (a) Sketch of the dual-wavelength in-vacuum cavity used to trap the atoms and to carry out the QND measurement. Atoms are trapped at the $813 \mathrm{~nm}$ intensity maxima represented in red, while they also interact with the nearest blue- and red-detuned cavity modes at $461 \mathrm{~nm}$ represented in blue and purple. (b) Simplified level scheme for Sr showing the $461 \mathrm{~nm}$ transition used for nondestructive detection and the $698 \mathrm{~nm}$ optical clock transition. (c) Diagram of the optical setup used for the QND measurement, and a sketch of the optical spectrum transmitted through the Mach-Zehnder interferometer (MZI). The six probe frequency components generated by the electro-optic modulator (EOM) chain are depicted in blue, interacting with the cavity modes in gray which surround the atomic transition in purple. The padlocks represent Pound-Drever-Hall loops used to stabilize the laser frequencies and the cavity lengths. 
of $S_{e e}$. From these two measurements, $S_{z}$ is calculated and the result is used to correct the LO frequency.

For the OLC in this work, we instead implement a QND measurement of the ground state population using the same optical cavity used to create the one-dimensional lattice trap. The cavity is coated to support optical modes surrounding the $461 \mathrm{~nm}$ transition from the ground to the auxiliary state. In the dispersive limit, where the detuning $\Delta$ of the cavity mode from the atomic transition is much larger than the cavity decay rate $(\kappa=2 \pi \times 330 \mathrm{kHz})$, the atomic decay rate $(\Gamma=2 \pi \times 30 \mathrm{MHz})$, and the vacuum Rabi frequency $2 g=2 \pi \times 680 \mathrm{kHz}$, the auxiliary state can be adiabatically eliminated. What remains is an effective coupling between the ground state population $S_{g g}$ and the photon number in the cavity mode $\hat{c}^{\dagger} \hat{c}$, described by the Hamiltonian [40,41]:

$$
\hat{H}_{c}=\hbar g^{2} \hat{c}^{\dagger} \hat{c} \hat{S}_{g g} / \Delta .
$$

This gives rise to an atom-induced frequency shift of the cavity resonance $\delta \nu=\left\langle\hat{H}_{c} / h\right\rangle /\left\langle\hat{c}^{\dagger} \hat{c}\right\rangle$. The basic principle of the QND measurement is to drive the cavity with a weak input field at $461 \mathrm{~nm}$, so that the reflected output field carries information about $\delta \nu$, and therefore acts as a meter for the number of atoms. The phase of the reflected field is measured destructively as a beat signal on a photodetector, giving a signal proportional to $S_{g g}$. To obtain $S_{z}$, which is needed to estimate the LO frequency error, the ground and excited state populations are swapped via a $\pi$ pulse $T$ at $698 \mathrm{~nm}$ and a second QND measurement of $S_{g g}$ is performed.

Further technical details of the QND measurement scheme [42] are outlined in Fig. 1. In order to provide first-order immunity to cavity length fluctuations $[25,43,44]$, we probe the difference in the atomic-induced frequency shift between two adjacent longitudinal cavity modes centered in frequency around the atomic transition. The optical field used to probe the two cavity modes is generated by sending the $461 \mathrm{~nm}$ laser through a Mach-Zehnder interferometer (MZI) amplitude modulator biased to zero throughput and driven at a frequency $\Omega / 2=2.09 \mathrm{GHz}$, matching the $4.18 \mathrm{GHz}$ free spectral range of the cavity. Additional sidebands at $\Omega / 2 \pm 125 \mathrm{MHz}$ are applied using the MZI modulator, generating strong frequency components which are directly reflected from the cavity input mirror. The strong directly reflected sidebands interfere with the cavity-coupled probe sidebands at $\pm \Omega / 2$, generating a Pound-Drever-Hall [45] beat signal at $125 \mathrm{MHz}$ proportional to the phase shift induced on the probe sidebands due to the atom-induced cavity shift $\delta \nu$.

\section{WEAK QND MEASUREMENT AND ATOM COHERENCE PRESERVATION}

To a good approximation the value of $S_{z}$ is conserved after the QND measurement, but other properties of the atomic system can be significantly altered. For example, a fundamental measurement backaction is exerted by photon shot noise in the probe beam, which generates an increase in the uncertainty of $S_{y}$ as we extract information about $S_{z}$, in compliance with the uncertainty principle $\Delta S_{y} \Delta S_{z} \geq$ $\left\langle\left|S_{x}\right|\right\rangle / 2$. In practice, however, two other technical effects are much larger for the QND scheme in this work: (1) the photon scatter $\Gamma_{\text {sc }}$ into free space and (2) the inhomogeneous ac Stark shift $\Delta_{\mathrm{ac}}$. Here, we discuss how these two forms of measurement backaction cause decay in the atom coherence $S_{x}$. We develop a model for the decoherence, and we present experimental data demonstrating weak QND readout of $S_{z}$ while preserving $S_{x}$ with $80 \%$ fidelity.

The scatter and the ac Stark shift depend on the radial position $\rho$ and the position $z$ along the cavity axis, according to

$$
\begin{gathered}
\Gamma_{\mathrm{sc}}(\rho, z)=\left\langle\Gamma_{\mathrm{sc}}(0, z)\right\rangle_{z} e^{-2 \rho^{2} / w_{0}^{2}}\left[\left(\cos ^{2} k z+\sin ^{2} k z\right)+\frac{2 \Delta_{\text {sum }} \Delta_{\text {diff }}}{\Delta_{\text {sum }}^{2}+\Delta_{\text {diff }}^{2}}\left(\cos ^{2} k z-\sin ^{2} k z\right)\right], \\
\Delta_{\mathrm{ac}}(\rho, z)=\left\langle\Gamma_{\mathrm{sc}}(0, z)\right\rangle_{z} e^{-2 \rho^{2} / w_{0}^{2}}\left[\frac{\Delta_{\text {diff }}}{\Gamma}\left(\cos ^{2} k z-\sin ^{2} k z\right)+\frac{\Delta_{\text {sum }}}{\Gamma}\left(\cos ^{2} k z+\sin ^{2} k z\right)\right]\left(1-\frac{2 \Delta_{\text {sum }}^{2}}{\Delta_{\text {sum }}^{2}+\Delta_{\text {diff }}^{2}}\right),
\end{gathered}
$$

where \langle\rangle$_{z}$ indicates a spatial average along $z, w_{0}=75 \mu \mathrm{m}$ is the waist of the cavity mode, $\Delta_{\text {diff }}=\left(\Delta_{\text {blue }}-\Delta_{\text {red }}\right) / 2=$ $2 \pi \times 2.09 \mathrm{GHz}$ is the average magnitude of the cavity mode detuning, $\Delta_{\text {sum }}=\left(\Delta_{\text {blue }}+\Delta_{\text {red }}\right) / 2=-2 \pi \times 173 \mathrm{MHz}$ is the asymmetry of the cavity mode detuning, $\Gamma=2 \pi \times$ $30 \mathrm{MHz}$ is the transition linewidth, and $k=2 \pi / \lambda$ is the wave number of the probe. In both equations we have explicitly written separate terms proportional to $\cos ^{2} k z$ and $\sin ^{2} k z$, created by the red- and blue-detuned probe sidebands respectively close to the center of the optical cavity. Ideally we would simplify the equations by choosing
$\Delta_{\text {sum }}=0$, but in practice a small offset is enforced by the technical constraint that the cavity length must be tuned to support a magic-wavelength $813 \mathrm{~nm}$ lattice to carry out high- $Q$ spectroscopy on the optical clock transition. Nonetheless, we still operate with $\Delta_{\text {diff }} \gg \Delta_{\text {sum }}$, such that Eq. (5) yields an approximately uniform photon scatter rate along $z$ while Eq. (6) yields an inhomogeneous ac Stark shift varying as $\cos 2 k z$.

In order to model the effect of $\Delta_{\mathrm{ac}}$ and $\Gamma_{\mathrm{sc}}$ on the collective atomic spin components $S_{i}$, we simulate a sample of a few thousand individual spins at different positions $\rho, z$ 
and propagate each spin using optical Bloch equations. The position $\rho$ of each atom is selected from a Gaussian distribution with standard deviation $\sigma_{\rho}=35 \mu \mathrm{m}$ corresponding to a radial temperature of $5 \mu \mathrm{K}$, which has been determined experimentally through sideband spectroscopy [46]. Since the radial trap frequency is only $120 \mathrm{~Hz}$, we treat $\rho$ as fixed throughout the QND measurement pulse, which has duration $t<0.5 \mathrm{~ms}$. The position of each atom along $z$ is randomly selected from one of 2000 sites of the $813 \mathrm{~nm}$ lattice trap, matching the experimentally measured width of the cloud. Along $z$, the trap frequency $63 \mathrm{kHz}$ is comparable to or faster than $1 / t$, so we make the approximation that the mean $z$ position of each atom is fixed to the center of the lattice site, but we average the scatter rate and ac Stark shift over a thermal waist $\sigma_{z}=50 \mathrm{~nm}$ corresponding to the $4 \mu \mathrm{K}$ measured axial temperature.

We investigate the QND probe backaction experimentally using the sequence depicted in Fig. 2(a). A sample of $6 \times 10^{3}$ atoms is first prepared in a coherent state with $\left\langle S_{x}\right\rangle=N / 2$ using a resonant $\pi / 2$ pulse from the clock laser. The QND probe is then applied for a variable amount of time $t$. After this, a second $\pi / 2$ pulse is applied from the clock laser, the phase of which is stepped by $0^{\circ}$ or $180^{\circ}$ with respect to the first pulse in order to map $S_{x}$ to $\pm S_{z}$. Finally, a destructive measurement is carried out of $S_{z}$, from which the value of $S_{x}$ just before the second $\pi / 2$ pulse can be inferred. To provide insensitivity to small systematic offsets in the $S_{z}$ measurement, the estimate of $S_{x}$ is based on the difference in measured $S_{z}$ between the two phases $0^{\circ}, 180^{\circ}$ of the final clock pulse. As observed in the data "without spin echo" in Fig. 2(b), the inhomogeneous ac Stark shift $\Delta_{\text {ac }}$ results in near-total loss of coherence at QND probe time $t=100 \mu \mathrm{s}$.
However, the rapid decoherence can be largely reversed using a spin-echo protocol. In the "with spin echo" sequence, an additional $\pi$ pulse is inserted with phase $90^{\circ}$ after the first QND probe, followed by a second QND probe. We observe that the decoherence from the ac Stark shift is strongly suppressed by the spin echo, with residual exponential decay of $S_{x}$ with a time constant $317 \mu$ s when using $125 \mathrm{fW}$ of cavity-coupled QND probe light. Since the $\pi$ pulse inverts the ground and excited population, the difference between the two QND probe signals in the spin-echo sequence provides a value for $S_{z}$. Therefore, a spin-echo QND probe sequence with a total probe time $t=60 \mu \mathrm{s}$ can act as a weak measurement of $S_{z}$, creating a signal to stabilize the clock LO while maintaining coherence with $80 \%$ fidelity.

\section{INCREASING THE $Q$ FACTOR VIA AN ATOM PHASE LOCK}

QND measurement in an OLC enables several novel applications that are otherwise impossible using conventional fluorescence readout techniques. Here we pursue one such application-the atom phase lock-in which the phase noise of the LO is tracked and corrected for via repeated weak measurement of the collective atomic spin. We characterize the performance of the APL to one OLC ( $\mathrm{Sr} 2$ [47]) using synchronous interrogation of a second OLC (Sr1 $[48,49])$ which has highly correlated sensitivity to fluctuations in the LO frequency and phase (see Fig. 3 and Supplemental Material [39]).

After loading approximately $1 \times 10^{4}$ atoms into the optical lattice, the APL is implemented in $\mathrm{Sr} 2$ following the scheme depicted in Fig. 4(a), which was originally
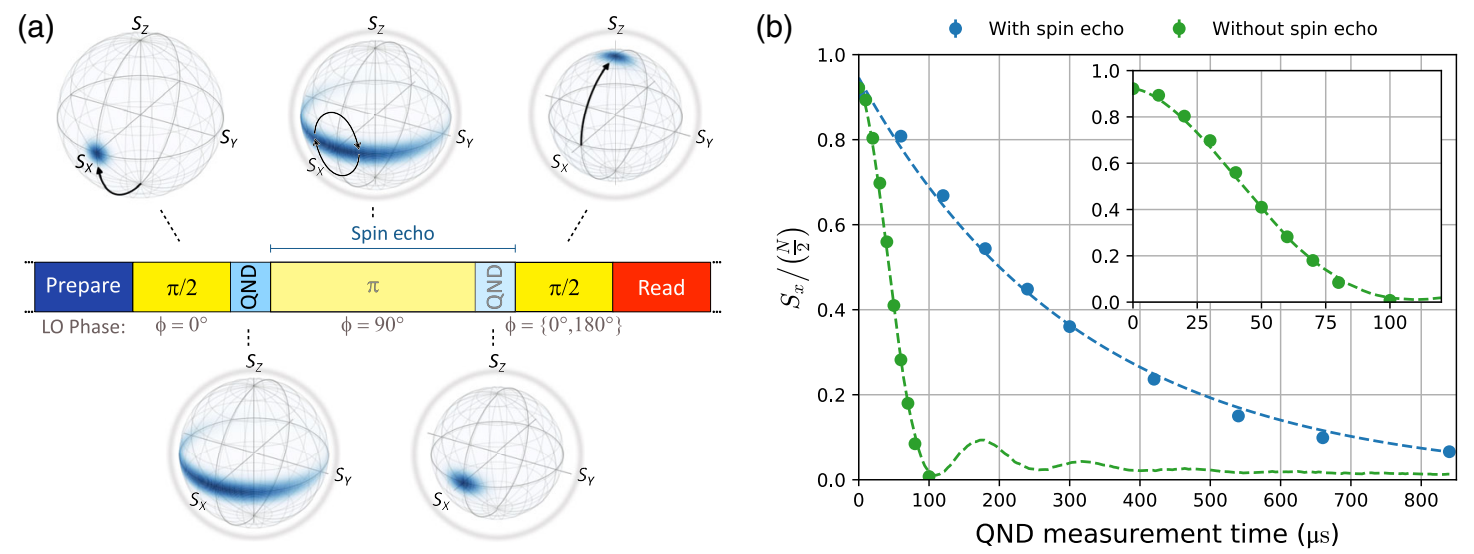

FIG. 2. Coherence preservation after the QND measurement. (a) Timing sequence used to measure coherence after QND measurement, and sketches of the atomic state in the Bloch sphere representation at each step of the sequence. The projection of atoms into the ground or excited state due to scattering of QND probe photons is represented by the shrinking radius of the Bloch sphere compared to its original size $N / 2$ (gray halo). The final Bloch sphere (top right) shows the case where the final $\pi / 2$-pulse phase is $\phi=0^{\circ}$. (b) The measured atomic coherence $S_{x}$ remaining after QND measurement as a function of total probe time. For the data with spin echo we use the total measurement time summed over the two QND probes, and fit an exponential decay with time constant $317 \mu$ s (blue dashed line). The model for coherence decay without spin echo (green dashed line, also displayed in the inset) is described in the main text. 
(a)

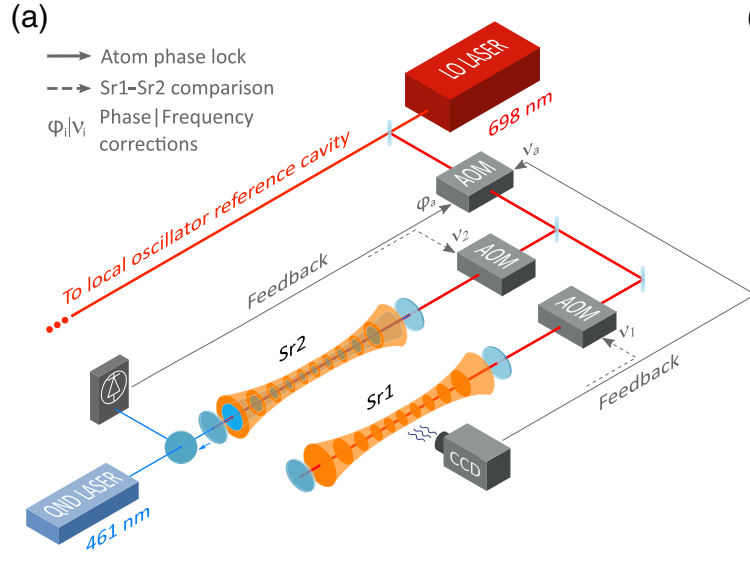

(b)

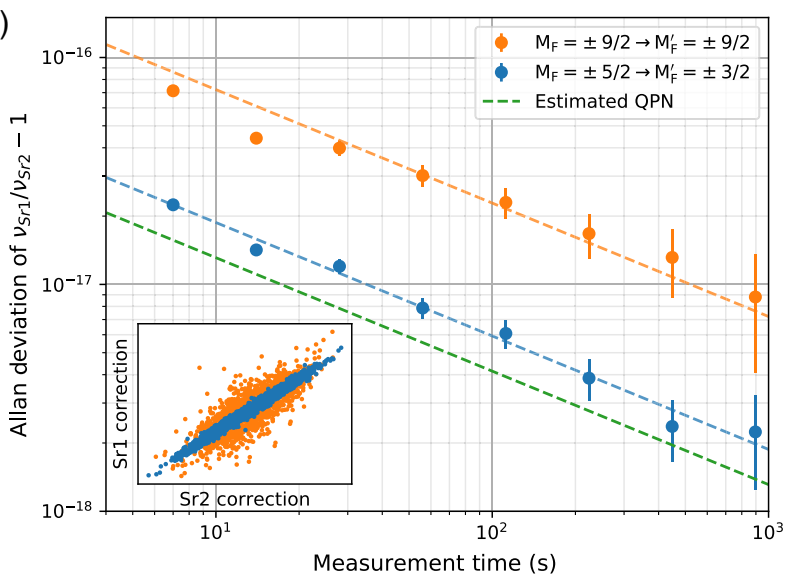

FIG. 3. Experimental setup and $\mathrm{Sr} 1-\mathrm{Sr} 2$ correlations. (a) LO light is distributed to $\mathrm{Sr} 1$ and $\mathrm{Sr} 2$ along separate optical paths, the lengths of which are actively stabilized. When the APL is engaged, phase corrections from $\mathrm{Sr} 2$ are applied to an acousto-optic modulator (AOM) shared by both systems, thereby increasing the coherence time of the light sent to $\mathrm{Sr} 1$. (b) Allan deviation of the frequency ratio between Sr1 and Sr2, with the APL disengaged and the OLCs independently stabilized using synchronous $300 \mathrm{~ms}$ Rabi pulses. Both clocks experience the same LO frequency fluctuations, resulting in highly correlated frequency corrections $\nu_{1}, \nu_{2}$. However, there are residual sources of noise-for example, linear Zeeman shift fluctuations, which are suppressed using a less sensitive Zeeman transition. After minimizing noise (see Supplemental Material [39]), the frequency instability approaches the quadrature sum of QPN from both clocks, $4 \times 10^{-17} / \sqrt{\tau}$. The cycle time is $1.75 \mathrm{~s}$ and atom numbers are $7 \times 10^{3}$ and $1.3 \times 10^{4}$ atoms in $\mathrm{Sr} 1$ and $\mathrm{Sr} 2$, respectively.

(a)

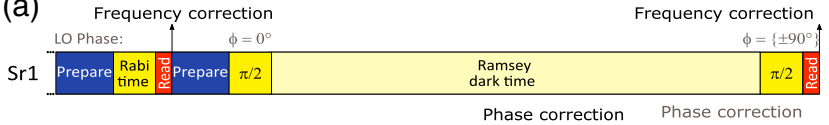

(b)

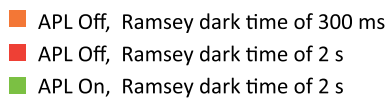

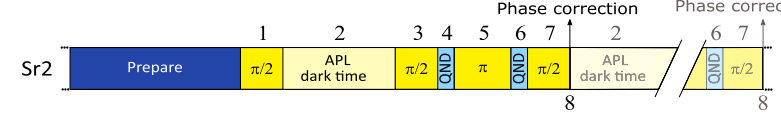
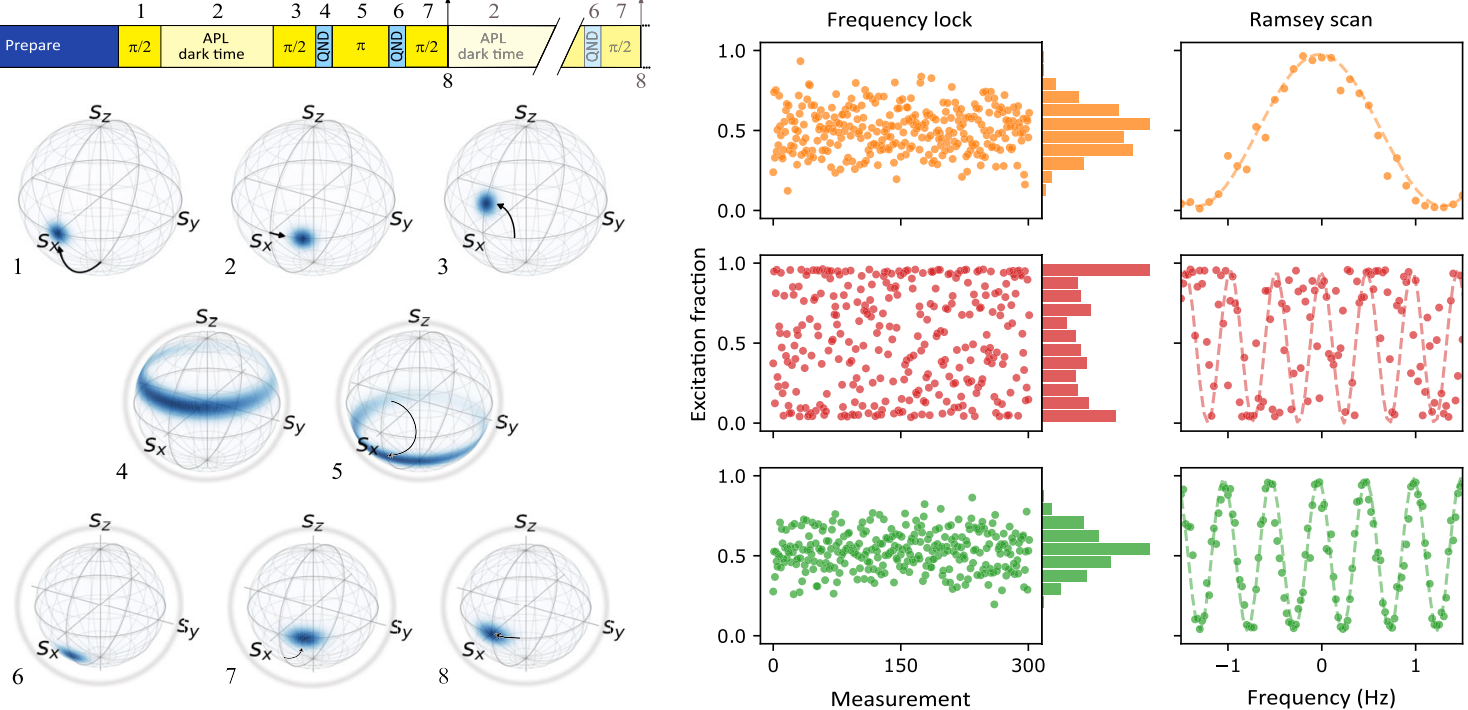

FIG. 4. Enhanced Ramsey spectroscopy via APL. (a) Timing sequence for the synchronous spectroscopy scheme, and Bloch sphere representation of the atomic state during the APL sequence. Atomic data from the Rabi time in Sr1 are used only when scanning over Sr1 Ramsey fringes. Prior to and during each scan, the Rabi data measure frequency drift of the free-running LO (typically between 0 and $3 \mathrm{mHz} \mathrm{s}^{-1}$ ), and allows us to apply LO drift compensation in a double-integrator control loop with attack time of approximately 100 clock cycles. (b) Results using Ramsey spectroscopy in $\mathrm{Sr} 1$, cointerrogated with $\mathrm{Sr} 2$ using the same LO. Left: excitation fractions with the LO frequency locked to the central Sr1 Ramsey fringe under three conditions: 300 ms Ramsey dark time with the APL to Sr2 disengaged (orange), $2 \mathrm{~s}$ dark time with the APL disengaged (red), and $2 \mathrm{~s}$ dark time with the APL engaged (green). Frequency scans over the Sr1 Ramsey fringes are shown on the right under the same conditions as above. With the APL engaged, the fringe width is measured to be $254(1) \mathrm{mHz}$ for a $2 \mathrm{~s}$ dark time. 
proposed [50] and demonstrated [51] for microwave atomic clocks. An initial $10 \mathrm{~ms} \pi / 2$ pulse drives the atomic ensemble into a coherent state on the equator of the Bloch sphere with $\left\langle S_{x}\right\rangle=N / 2$. As in a normal Ramsey sequence, the atomic state is left to freely evolve during which time it accumulates a phase shift relative to the LO. In the small angle approximation the accumulated LO phase is proportional to $\left\langle S_{y}\right\rangle$, which is read out in the following procedure: a $\pi / 2$ pulse is driven by the $\mathrm{LO}$, the phase of which is stepped by $90^{\circ}$ with respect to the initial pulse in order to map $S_{y}$ to $S_{z}$. The ground state atom number $S_{g g}$ is then read out via a QND measurement pulse with duration $t=30 \mu \mathrm{s}$. To read out the excited state atom number $S_{e e}$, a $\pi$ pulse is driven with LO phase $-90^{\circ}$ relative to the initial pulse, before a second QND measurement is applied for $t=30 \mu \mathrm{s}$. Finally, the LO phase is stepped again to $90^{\circ}$ and a final $\pi / 2$ pulse is applied to return the collective atomic spin to the equator of the Bloch sphere. Based on the results of the two QND measurements, the LO phase is stepped to align the atomic spin to point along the $x$ axis of the Bloch sphere. Repeating the free-evolution time and the QND measurement procedure several times in succession, a phase lock of the LO to the atomic transition can be maintained for several seconds-well beyond the coherence time of the free-running LO.

To characterize the improvement in LO phase noise, the Sr2-phase-stabilized light is used to interrogate Sr1, with results shown in Fig. 4(b). Atomic samples are prepared in parallel in both systems and probed synchronously using the same local oscillator. Sr2 is used to implement the APL while $\mathrm{Sr} 1$ performs standard Ramsey spectroscopy. To get a baseline measurement of the free-running laser phase noise, the Sr2 APL is first disengaged and Sr1 is operated as a clock with Ramsey spectroscopy dark time $T=300 \mathrm{~ms}$. When we lock the frequency of the LO to the central Sr1 fringe, we observe noise in the in-lock excitation fraction corresponding to a standard deviation for the accumulated LO phase error of $290 \mathrm{mrad}$. Increasing Ramsey dark time to $2 \mathrm{~s}$, but with the APL still disengaged, shows that the accumulated phase error is too large to operate the clock reliably. This is clear from the S-shaped histogram of the excitation noise in Fig. 4, indicating the phase error is well outside the $\pm \pi / 2$ range that is needed to determine unambiguously the average frequency offset during the dark time. A final dataset is taken with the APL engaged on Sr2 during the 2 s Ramsey dark time in Sr1. Specifically, the APL consists of five repetitions of a $340 \mathrm{~ms}$ dark time followed by $60 \mathrm{~ms}$ QND phase measurement and correction. The phase corrections are applied onto an acoustooptic modulator (AOM) which corrects the LO light prior to it being split and sent to both clocks. Therefore, the average residual phase error accumulated during the APL can be characterized based on the excitation noise in Sr1 when locked to the clock transition, and was determined to be 240 mrad. Finally, a scan of the full Ramsey fringe in $\mathrm{Sr} 1$ shows no degradation of the $95 \%$ contrast and a Fourier limited linewidth of $254(1) \mathrm{mHz}$, corresponding to an oscillator $Q$ factor of $1.7 \times 10^{15}$. This is within a factor of 3 of the finest scan resolution achieved using state-of-the-art LOs, but unlike earlier high-resolution scans $[28,52,53]$ we observe no significant loss of contrast on the Ramsey fringes. To our knowledge this matches the narrowest spectroscopic feature to which any oscillator has yet been stabilized [54]. Extending the APL time further, either through longer free-evolution time or increased number of QND measurements, resulted in increased phase noise in $\mathrm{Sr} 1$.

\section{CONCLUSION}

We have demonstrated that the QND-based APL is an effective approach to improve the phase coherence of an ultrastable laser, making it a competitive alternative to other strategies for minimizing the technical noise of the LO [55]. Increasing the LO phase coherence directly impacts the frequency stability performance of the OLC, as it enables longer Ramsey dark time $T$, resulting in an increased $Q$ factor and a steeper discriminant of the atomic excitation fraction against the LO frequency. The clearest impact of this is on the quantum-projection-noise- (QPN) induced fractional frequency instability, which for spectroscopy of $N$ atoms with a signal contrast $C$ and a cycle time of $T_{c}$ is given by

$$
\sigma_{\mathrm{QPN}}(\tau)=\frac{1}{\pi Q C} \sqrt{\frac{T_{c}}{N \tau}}
$$

where $\sigma(\tau)$ denotes the Allan deviation for averaging time $\tau$ in seconds. Specifically for Sr1, which operates with $5 \times 10^{3}$ atoms, the sevenfold increase in the $Q$ factor achieved by extending the Ramsey probe time from $300 \mathrm{~ms}$ to $2 \mathrm{~s}$, with corresponding cycle times 1.3 and $3 \mathrm{~s}$ respectively, reduces the QPN instability from $2.1 \times$ $10^{-17} / \sqrt{\tau}$ to $4.8 \times 10^{-18} / \sqrt{\tau}$.

Another important source of instability in OLCs is the Dick effect, caused by short-term LO frequency noise which is sampled by dead time (primarily cooling time) in the clock sequence. For Ramsey spectroscopy, in the limit of instantaneous $\pi / 2$ pulses, the Dick-effect instability is given by [56]

$$
\sigma_{\text {Dick }}(\tau)=\sqrt{\frac{1}{\tau} \sum_{k=1}^{\infty} S_{y}\left(k / T_{c}\right)\left[\frac{\sin \left(\pi k T / T_{c}\right)}{\pi k T / T_{c}}\right]^{2}} .
$$

Increasing the ratio of the Ramsey dark time to the cycle time helps to suppress this effect. Estimating the precise reduction in Dick-effect instability is complex, as it depends on the power spectral density $S_{y}(f)$ of the fractional frequency fluctuations of the $\mathrm{LO}$ at harmonics of the cycle frequency. Under the assumption that LO flicker noise, 
which we have directly measured to be $8 \times 10^{-17}$, is the dominant noise process, the estimated Dick-effect induced instability for a $2 \mathrm{~s}$ Ramsey dark time is $5 \times 10^{-17} / \sqrt{\tau}$ - a factor of 1.6 below what is expected for a $300 \mathrm{~ms}$ Ramsey dark time, leading to a reduction in measurement time by a factor of 2.5 to reach the same precision. In the future, an optical frequency comb could transfer the enhanced phased stability of the LO to other wavelengths in order to improve the performance of optical clocks based on different atomic species [57,58]. In particular, applying this technique to $\mathrm{Yb}^{+}$ or highly charged ion clocks which are limited by QPN, but exhibit a large sensitivity to changes in the fine structure constant, could facilitate improved tests of fundamental physics [59-61].

It is instructive to compare our QND-based method against alternative approaches to extend the coherence time of the LO laser. In one demonstration, the LO was prestabilized to an OLC with $50 \%$ duty cycle, and then used to interrogate a second OLC [28]. However, with this approach the attainable extension of probe time is limitedthere is still considerable dead time of several hundred milliseconds needed to cool atoms in the prestabilization OLC, during which the phase of the LO is going unmeasured. Another recent demonstration used a novel multipulse interrogation scheme in an OLC to achieve dynamical decoupling of the laser phase noise. The dynamical decoupling allows the OLC to coarsely track the laser phase for a continuous spectroscopy time much longer than the coherence of the LO [62]. However, the dynamical decoupling also reduces the mean sensitivity of the OLC to laser phase fluctuations, resulting in a higher sensitivity to quantum projection noise compared with the QND-based scheme presented in this work. Finally, another promising alternative would be to make use of recent advances combining strontium atoms and tweezer arrays [53,63]. Such platforms allow for repeated probing of the clock transition and detection, in some cases up to 15 times, without needing to reload the atoms. However, since these experiments rely on fluorescence detection, the phase coherence between the $\mathrm{LO}$ and the atoms is lost during detection. If repeated fluorescence readout in a tweezer array were used to implement a destructive form of the atom phase lock, the phase measurement errors (e.g., from quantum projection noise) would accumulate with each interrogation pulse. In contrast, the QND measurementbased approach preserves coherence after each measurement, resulting in phase noise in earlier measurements being corrected for by subsequent measurements.

Considering alternative applications of the APL scheme, we speculate that it could help to enable high- $Q$ spectroscopy in environments where the ultimate performance of cavity stabilized lasers cannot be reached, for instance in field deployed systems. At the same time, the QND measurement scheme underpinning the APL also opens the door to other configurations of quantum-enhanced optical atomic clocks. Going forward, it will be instructive to characterize the QND measurement scheme in more detail, for example, by using colder atomic samples in a bettercontrolled motional state. Relative to the data presented in Fig. 2, we observe that the QND contrast decay rate can be reduced by a factor of approximately 2 by adjusting the MZI setup in Fig. 1 so that the two electro-optic modulators (EOMs) generating the stronger probe sidebands at $\Omega / 2 \pm$ $125 \mathrm{MHz}$ are placed on different arms of the MZI-this eliminates spurious second-order frequency components near atomic resonance created at the difference frequency between those EOMs, thereby mitigating a source of excess scattered photons. If the QND readout noise and measurement backaction can be controlled close to their shot noise limits, our quantum nondemolition measurement apparatus could be used to generate squeezed states with reduced QPN, offering a route toward OLC comparison with unprecedented frequency precision. Finally, the ability to engineer squeezing in Sr could also have implications beyond precision timekeeping, for example, by improving the performance of $\mathrm{Sr}$ atom interferometers [64] toward the sensitivity necessary to observe gravitational waves [65].

\section{ACKNOWLEDGMENTS}

This work was financially supported by the UK Department for Business, Energy and Industrial Strategy as part of the National Measurement System Programme, and by the European Metrology Programme for Innovation and Research (EMPIR) Project No. 17FUN03-USOQS. This project has received funding from the EMPIR programme cofinanced by the Participating States and from the European Union's Horizon 2020 research and innovation programme. A. V. acknowledges funding from the Engineering and Physical Sciences Research Council (EPSRC UK) through the Controlled Quantum Dynamics Centre for Doctoral Training (EP/L016524/1) for the core duration of this work. We thank Helen Margolis, Alissa Silva, and Jake Paterson for operating the optical frequency comb, and Rachel Godun and Rich Hendricks for careful reading of the manuscript.

[1] V. B. Braginsky, Y. I. Vorontsov, and K. S. Thorne, Quantum Non-Demolition Measurements, Science 209, 547 (1980).

[2] N. Ofek, A. Petrenko, R. Heeres, P. Reinhold, Z. Leghtas, B. Vlastakis, Y. Liu, L. Frunzio, S. M. Girvin, L. Jiang, M. Mirrahimi, M. H. Devoret, and R. J. Schoelkopf, Extending the Lifetime of a Quantum Bit with Error Correction in Superconducting Circuits, Nature (London) 536, 441 (2016).

[3] U. Vool, S. Shankar, S. O. Mundhada, N. Ofek, A. Narla, K. Sliwa, E. Zalys-Geller, Y. Liu, L. Frunzio, R. J. Schoelkopf, S.M. Girvin, and M.H. Devoret, Continuous Quantum 
Non-Demolition Measurement of the Transverse Component of a Qubit, Phys. Rev. Lett. 117, 133601 (2016).

[4] S. Hacohen-Gourgy, L. S. Martin, E. Flurin, V. V. Ramasesh, K. B. Whaley, and I. Siddiqi, Quantum Dynamics of Simultaneously Measured Non-Commuting Observables, Nature (London) 538, 491 (2016).

[5] M. Raha, S. Chen, C. M. Phenicie, S. Ourari, A. M. Dibos, and J.D. Thompson, Optical Quantum Non-Demolition Measurement of a Single Rare Earth Ion Qubit, Nat. Commun. 11, 1605 (2020).

[6] T. Nakajima, A. Noiri, J. Yoneda, M. R. Delbecq, P. Stano, T. Otsuka, K. Takeda, S. Amaha, G. Allison, K. Kawasaki et al., Quantum Non-Demolition Measurement of an Electron Spin Qubit, Nat. Nanotechnol. 14, 555 (2019).

[7] X. Xue, B. D'Anjou, T. F. Watson, D. R. Ward, D. E. Savage, M. G. Lagally, M. Friesen, S. N. Coppersmith, M. A. Eriksson, W. A. Coish, and L. M. K. Vandersypen, Repetitive Quantum Non-Demolition Measurement and Soft Decoding of a Silicon Spin Qubit, Phys. Rev. X 10, 021006 (2020).

[8] F. Lecocq, J. B. Clark, R. W. Simmonds, J. Aumentado, and J. D. Teufel, Quantum Non-Demolition Measurement of a Nonclassical State of a Massive Object, Phys. Rev. X 5, 041037 (2015).

[9] M. Rossi, D. Mason, J. Chen, Y. Tsaturyan, and A. Schliesser, Measurement-Based Quantum Control of Mechanical Motion, Nature (London) 563, 53 (2018).

[10] S. Kono, K. Koshino, Y. Tabuchi, A. Noguchi, and Y. Nakamura, Quantum Non-Demolition Detection of an Itinerant Microwave Photon, Nat. Phys. 14, 546 (2018).

[11] A. Reiserer, S. Ritter, and G. Rempe, Non-Destructive Detection of an Optical Photon, Science 342, 1349 (2013).

[12] J.-C. Besse, S. Gasparinetti, M. C. Collodo, T. Walter, P. Kurpiers, M. Pechal, C. Eichler, and A. Wallraff, SingleShot Quantum Non-Demolition Detection of Individual Itinerant Microwave Photons, Phys. Rev. X 8, 021003 (2018).

[13] M. Brune, E. Hagley, J. Dreyer, X. Maître, A. Maali, C. Wunderlich, J. M. Raimond, and S. Haroche, Observing the Progressive Decoherence of the Meter in a Quantum Measurement, Phys. Rev. Lett. 77, 4887 (1996).

[14] J. Cramer, N. Kalb, M. A. Rol, B. Hensen, M. S. Blok, M. Markham, D. J. Twitchen, R. Hanson, and T. H. Taminiau, Repeated Quantum Error Correction on a Continuously Encoded Qubit by Real-Time Feedback, Nat. Commun. 7, 11526 (2016).

[15] D. B. Hume, T. Rosenband, and D. J. Wineland, HighFidelity Adaptive Qubit Detection through Repetitive Quantum Non-Demolition Measurements, Phys. Rev. Lett. 99, 120502 (2007).

[16] F. Wolf, Y. Wan, J. C. Heip, F. Gebert, C. Shi, and P. O. Schmidt, Non-Destructive State Detection for Quantum Logic Spectroscopy of Molecular Ions, Nature (London) 530, 457 (2016).

[17] V. Shah, G. Vasilakis, and M. V. Romalis, High Bandwidth Atomic Magnetometery with Continuous Quantum NonDemolition Measurements, Phys. Rev. Lett. 104, 013601 (2010).

[18] G. Colangelo, F. M. Ciurana, L. C. Bianchet, R. J. Sewell, and M. W. Mitchell, Simultaneous Tracking of Spin Angle and Amplitude beyond Classical Limits, Nature (London) 543, 525 (2017).

[19] O. Hosten, N. J. Engelsen, R. Krishnakumar, and M. A. Kasevich, Measurement Noise 100 Times Lower than the Quantum-Projection Limit Using Entangled Atoms, Nature (London) 529, 505 (2016).

[20] A. Louchet-Chauvet, J. Appel, J. J. Renema, D. Oblak, N. Kjaergaard, and E. S. Polzik, Entanglement-Assisted Atomic Clock beyond the Projection Noise Limit, New J. Phys. 12, 065032 (2010).

[21] A. Kuzmich, L. Mandel, and N. P. Bigelow, Generation of Spin Squeezing via Continuous Quantum Non-Demolition Measurement, Phys. Rev. Lett. 85, 1594 (2000).

[22] K. C. Cox, G. P. Greve, J. M. Weiner, and J. K. Thompson, Deterministic Squeezed States with Collective Measurements and Feedback, Phys. Rev. Lett. 116, 093602 (2016).

[23] M. H. Schleier-Smith, I. D. Leroux, and V. Vuletić, States of an Ensemble of Two-Level Atom with Reduced Quantum Uncertainty, Phys. Rev. Lett. 104, 073604 (2010).

[24] B. Braverman, A. Kawasaki, E. Pedrozo-Peñafiel, S. Colombo, C. Shu, Z. Li, E. Mendez, M. Yamoah, L. Salvi, D. Akamatsu, Y. Xiao, and V. Vuletić, Near-Unitary Spin Squeezing in ${ }^{171} \mathrm{Yb}$, Phys. Rev. Lett. 122, 223203 (2019).

[25] G. Vallet, E. Bookjans, U. Eismann, S. Bilicki, R. L. Targat, and J. Lodewyck, A Noise-Immune Cavity-Assisted NonDestructive Detection for an Optical Lattice Clock in the Quantum Regime, New J. Phys. 19, 083002 (2017).

[26] M. A. Norcia and J. K. Thompson, Strong Coupling on a Forbidden Transition in Strontium and Non-Destructive Atom Counting, Phys. Rev. A 93, 023804 (2016).

[27] E. Oelker, R. B. Hutson, C. J. Kennedy, L. Sonderhouse, T. Bothwell, A. Goban, D. Kedar, C. Sanner, J. M. Robinson, G. E. Marti, D. G. Matei, T. Legero, M. Giunta, R. Holzwarth, F. Riehle, U. Sterr, and J. Ye, Demonstration of $4.8 \times 10^{-17}$ Stability at $1 \mathrm{~s}$ for Two Independent Optical Clocks, Nat. Photonics 13, 714 (2019).

[28] M. Schioppo, R. C. Brown, W. F. McGrew, N. Hinkley, R. J. Fasano, K. Beloy, T. H. Yoon, G. Milani, D. Nicolodi, J. Sherman et al., Ultrastable Optical Clock with Two ColdAtom Ensembles, Nat. Photonics 11, 48 (2017).

[29] I. Ushijima, M. Takamoto, M. Das, T. Ohkubo, and H. Katori, Cryogenic Optical Lattice Clocks, Nat. Photonics 9 , 185 (2015).

[30] W. F. McGrew, X. Zhang, R. J. Fasano, S. A. Schäffer, K. Beloy, D. Nicolodi, R. C. Brown, N. Hinkley, G. Milani, M. Schioppo, T. H. Yoon, and A. D. Ludlow, Atomic Clock Performance Enabling Geodesy below the Centimetre Level, Nature (London) 564, 87 (2018).

[31] J. Lodewyck, On a Definition of the SI Second with a Set of Optical Clock Transitions, Metrologia 56, 055009 (2019).

[32] J. Grotti et al., Geodesy and Metrology with a Transportable Optical Clock, Nat. Phys. 14, 437 (2018).

[33] T. Takano, M. Takamoto, I. Ushijima, N. Ohmae, T. Akatsuka, A. Yamaguchi, Y. Kuroishi, H. Munekane, B. Miyahara, and H. Katori, Geopotential Measurements with Synchronously Linked Optical Lattice Clocks, Nat. Photonics 10, 662 (2016).

[34] P. Wcisło et al., New Bounds on Dark Matter Coupling from a Global Network of Optical Atomic Clocks, Sci. Adv. 4, eaau4869 (2018). 
[35] P. Delva et al., Test of Special Relativity Using a Fiber Network of Optical Clocks, Phys. Rev. Lett. 118, 221102 (2017).

[36] M. Takamoto, I. Ushijima, N. Ohmae, T. Yahagi, K. Kokado, H. Shinkai, and H. Katori, Test of General Relativity by a Pair of Transportable Optical Lattice Clocks, Nat. Photonics 14, 411 (2020).

[37] B. M. Roberts et al., Search for Transient Variations of the Fine Structure Constant and Dark Matter Using FiberLinked Optical Atomic Clocks, New J. Phys. 22, 093010 (2020).

[38] J. Ye, H. Kimble, and H. Katori, Quantum State Engineering and Precision Metrology Using State-Insensitive Light Traps, Science 320, 1734 (2008).

[39] See Supplemental Material at http://link.aps.org/ supplemental/10.1103/PhysRevX.10.041052 for details of the local oscillator, the science packages and the weak measurement apparatus, and for the techniques used to minimize differential phase noise between $\mathrm{Sr} 1$ and $\mathrm{Sr} 2$.

[40] A. Blais, R.-S. Huang, A. Wallraff, S. M. Girvin, and R. J. Schoelkopf, Cavity Quantum Electrodynamics for Superconducting Electrical Circuits: An Architecture for Quantum Computation, Phys. Rev. A 69, 062320 (2004).

[41] D. Zueco, G. M. Reuther, S. Kohler, and P. Hänggi, QubitOscillator Dynamics in the Dispersive Regime: Analytical Theory beyond the Rotating-Wave Approximation, Phys. Rev. A 80, 033846 (2009).

[42] R. Hobson, W. Bowden, A. Vianello, I. R. Hill, and P. Gill, Cavity-Enhanced Non-Destructive Detection of Atoms for an Optical Lattice Clock, Opt. Express 27, 37099 (2019).

[43] R. Long, A. Tuchman, and M. Kasevich, Multiple Frequency Modulation for Low-Light Atom Measurements in an Optical Cavity, Opt. Lett. 32, 2502 (2007).

[44] J. Ye, L.-S. Ma, and J. L. Hall, Ultrasensitive Detections in Atomic and Molecular Physics: Demonstration in Molecular Overtone Spectroscopy, J. Opt. Soc. Am. B 15, 6 (1998).

[45] R. W. P. Drever, J. L. Hall, F. V. Kowalski, J. Hough, G. M. Ford, A. J. Munley, and H. Ward, Laser Phase and Frequency Stabilization Using an Optical Resonator, Appl. Phys. B 31, 97 (1983).

[46] S. Blatt, J. W. Thomsen, G. K. Campbell, A. D. Ludlow, M. D. Swallows, M. J. Martin, M. M. Boyd, and J. Ye, Rabi Spectroscopy and Excitation Inhomogeneity in a OneDimensional Optical Lattice Clock, Phys. Rev. A 80 , 052703 (2009).

[47] W. Bowden, R. Hobson, I. R. Hill, A. Vianello, M. Schioppo, A. Silva, H. S. Margolis, P. E. G. Baird, and P. Gill, A Pyramid MOT with Integrated Optical Cavities as a Cold Atom Platform for an Optical Lattice Clock, Sci. Rep. 9, 1 (2019).

[48] I. R. Hill, R. Hobson, W. Bowden, E. M. Bridge, S. Donnellan, E. A. Curtis, and P. Gill, A Low Maintenance Sr Optical Lattice Clock, J. Phys. Conf. Ser. 723, 012019 (2016).

[49] R. Hobson, W. Bowden, A. Vianello, A. Silva, C. F. A. Baynham, H. S. Margolis, P. E. G. Baird, P. Gill, and I. R. Hill, A Strontium Optical Lattice Clock with $1 \times 10^{-17}$ Uncertainty and Measurement of Its Absolute Frequency, Metrologia 57, 065026 (2020).
[50] N. Shiga and M. Takeuchi, Locking the Local Oscillator Phase to the Atomic Phase via Weak Measurement, New J. Phys. 14, 023034 (2012).

[51] R. Kohlhaas, A. Bertoldi, E. Cantin, A. Aspect, A. Landragin, and P. Bouyer, Phase Locking a Clock Oscillator to a Coherent Atomic Ensemble, Phys. Rev. X 5, 021011 (2015).

[52] S. L. Campbell, R. B. Hutson, G. E. Marti, A. Goban, N. D. Oppong, R. L. McNally, L. Sonderhouse, J. M. Robinson, W. Zhang, B. J. Bloom, and J. Ye, A Fermi-Degenerate Three-Dimensional Optical Lattice Clock, Science 358, 90 (2017).

[53] M. A. Norcia, A. W. Young, W. J. Eckner, E. Oelker, J. Ye, and A. M. Kaufman, Seconds-Scale Coherence on an Optical Clock Transition in a Tweezer Array, Science 366, 93 (2019).

[54] S. Origlia, M. S. Pramod, S. Schiller, Y. Singh, K. Bongs, R. Schwarz, A. Al-Masoudi, S. Dörscher, S. Herbers, S. Häfner, U. Sterr, and C. Lisdat, Towards an Optical Clock for Space: Compact, High-Performance Optical Lattice Clock Based on Bosonic Atoms, Phys. Rev. A 98, 053443 (2018).

[55] D. G. Matei, T. Legero, S. Häfner, C. Grebing, R. Weyrich, W. Zhang, L. Sonderhouse, J. M. Robinson, J. Ye, F. Riehle, and U. Sterr, $1.5 \mu \mathrm{m}$ Lasers with Sub-10 $\mathrm{mHz}$ Linewidth, Phys. Rev. Lett. 118, 263202 (2017).

[56] G. Santarelli, C. Audoin, A. Makdissi, P. Laurent, G. J. Dick, and A. Clairon, Frequency Stability Degradation of an Oscillator Slaved to a Periodically Interrogated Atomic Resonator, IEEE Trans. Ultrason. Ferroelectr. Freq. Control 45, 887 (1998)

[57] M. Giunta, W. Hänsel, M. Fischer, M. Lezius, T. Udem, and R. Holzwarth, Real-Time Phase Tracking for Wide-Band Optical Frequency Measurements at the 20th Decimal Place, Nat. Photonics 14, 44 (2020).

[58] E. Benkler, B. Lipphardt, T. Puppe, R. Wilk, F. Rohde, and U. Sterr, End-to-End Topology for Fiber Comb Based Optical Frequency Transfer at the $10^{-21}$ Level, Opt. Express 27, 36886 (2019).

[59] R. M. Godun, P. B. R. Nisbet-Jones, J. M. Jones, S. A. King, L. A. M. Johnson, H. S. Margolis, K. Szymaniec, S. N. Lea, K. Bongs, and P. Gill, Frequency Ratio of Two Optical Clock Transitions in ${ }^{171} \mathrm{Yb}^{+}$and Constraints on the Time Variation of Fundamental Constants, Phys. Rev. Lett. 113, 210801 (2014).

[60] N. Huntemann, B. Lipphardt, C. Tamm, V. Gerginov, S. Weyers, and E. Peik, Improved Limit on a Temporal Variation of $m_{p} / m_{e}$ from Comparisons of $\mathrm{Yb}^{+}$ and Cs Atomic Clocks, Phys. Rev. Lett. 113, 210802 (2014).

[61] M. G. Kozlov, M. S. Safronova, J. R. C. López-Urrutia, and P. O. Schmidt, Highly Charged Ions: Optical Clocks and Applications in Fundamental Physics, Rev. Mod. Phys. 90, 045005 (2018).

[62] S. Dörscher, A. Al-Masoudi, M. Bober, R. Schwarz, R. Hobson, U. Sterr, and C. Lisdat, Dynamical Decoupling of Laser Phase Noise in Compound Atomic Clocks, Commun. Phys. 3, 185 (2020).

[63] I. S. Madjarov, A. Cooper, A. L. Shaw, J. P. Covey, V. Schkolnik, T. H. Yoon, J. R. Williams, and M. Endres, An 
Atomic-Array Optical Clock with Single-Atom Readout, Phys. Rev. X 9, 041052 (2019).

[64] L. Hu, N. Poli, L. Salvi, and G. M. Tino, Atom Interferometry with the Sr Optical Clock Transition, Phys. Rev. Lett. 119, 263601 (2017).
[65] P. W. Graham, J. M. Hogan, M. A. Kasevich, and S. Rajendran, New Method for Gravitational Wave Detection with Atomic Sensors, Phys. Rev. Lett. 110, 171102 (2013). 